\title{
PALAEOENVIRONMENTAL SIGNIFICANCE OF ALLOCHTHONOUS VS. AUTOCHTHONOUS LATE QUATERNARY OSTRACODES FROM IMARUÍ LAGOON AND D'UNA RIVER, SOUTHERN BRAZIL
}

\author{
JOÃO CARLOS COIMBRA \\ Departamento de Paleontologia e Estratigrafia, Instituto de Geociências, UFRGS, Cx.P. 15001, 91501-970, Porto Alegre, \\ RS, Brazil. joao.coimbra@ufrgs.br \\ KAREN BADARACO COSTA \\ Laboratório de Paleoceanografia do Atlântico Sul, Instituto Oceanográfico, USP, Praça do Oceanográfico, 191, \\ 05508-900, São Paulo, SP, Brazil. costa.karen@gmail.br \\ GERSON FAUTH \\ Laboratório de Micropaleontologia, PPGeo, UNISINOS, Av. Unisinos, 950, 93022-000, São Leopoldo, RS, Brazil. \\ gersonf@unisinos.br
}

\begin{abstract}
Late Quaternary ostracodes collected from 15 cores in two adjacent localities, Imaruí and D'Una River, both in Santa Catarina State, southern Brazil, were analyzed. Among the 12 identified species, only three are left in open nomenclature. The population age-structure was studied in order to distinguish between allochthonous vs. autochthonous ostracodes in each sample. Two typically mixohaline species, Cyprideis multidentata Hartmann and $C$. salebrosa Bold, are the most abundant autochthonous fossils in the cores. The taphonomic analysis and the sedimentological evidences allow the inference of a permanent lagoonal palaeoenvironment in the 15 cores. Micropalaeontological and geological data suggest that the marine species were transported into the lagoonal palaeoenvironment, reworked with the autochthonous mixohaline fauna and deposited along the lagoonal border.
\end{abstract}

Key words: Late Quaternary, ostracodes, palaeoenvironments, taphonomy, southern Brazil.

\begin{abstract}
RESUMO - Foram analisados os ostracodes recuperados de 15 perfurações realizadas em duas áreas adjacentes, o município de Imaruí e a região do Rio D’uma, ambas no Estado de Santa Catarina, sul do Brasil. Dentre as 12 espécies identificadas apenas três foram deixadas em nomenclatura aberta. O estudo da estrutura populacional, realizado em cada amostra, permitiu a diferenciação entre fauna alóctone $v s$. autóctone. As espécies mixohalinas Cyprideis multidentata Hartmann e C. salebrosa Bold são os fósseis autóctones mais abundantes nos testemunhos investigados. A análise tafonômica e as evidências sedimentológicas indicaram um paleoambiente tipicamente lagunar ao longo das 15 perfurações. Tanto a microfauna quanto os dados advindos da geologia sugerem que as espécies marinhas foram transportadas para o ambiente lagunar, retrabalhadas juntamente com a fauna mixohalina e depositadas ao longo da borda da laguna.
\end{abstract}

Palavras-chave: Neoquaternário, ostracodes, paleoambientes, tafonomia, sul do Brasil.

\section{INTRODUCTION}

The coast of Santa Catarina State, southern of Brazil, is characterized by extensive sedimentary coastal plains with mineral deposits. The region has a large deposit of calcareous shells with economic relevance and great significance in an evolutionary perspective of the coastal plain. The southern coast of this State has some lagoons and coastal lakes being the Mirim, Imaruí and Santo Antônio lagoons complex the main regional aquatic body, which has been object of geological, sedimentological and morphological studies (Caruso Jr., 1995a). The shell deposits have been studied from a palaeontological point of view, especially concerning micromolluscs (Pitoni, 1993; Mendes, 1993) and foraminifers (Thiesen et al., 1993). The previous palaeontological studies were mainly taxonomic ones included some data about palaeoecology. Using sedimentological analysis Caruso Jr. (1995a, 1995b) dated the shell deposits in the Imaruí region to the Holocene.

\section{STUDY AREA}

Studies carried out on the coast of the State of Santa Catarina have revealed large oscillations of the sea level during the Quaternary (Suguio et al., 1985, 2005; Martin \& Suguio, 
1986; Martin et al., 1988; Gré et al., 1993). During that interval, the evolution of the southern Brazilian continental margin was driven by global changes affecting climate and sea level oscillations, due to the geode response to the variations of the ice masses and surface water distribution. In particular, the sea level changes exerted the strongest influence on the construction of the coastal plains, and marine sediments deposited on the continental deposits were repeatedly reworked by transgressions/regressions (Caruso Jr., 1995a, 1995b, 1999; Villwock \& Tomazelli, 1995; Carreño et al., 1999; Caruso Jr. et al., 1999). This allowed the accumulation of coastal marine and eolic deposits which, in some cases, originated bars that isolated coastal lagoons. The shell deposits have an irregular distribution along the coastal plain probably associated to a palaeolagoonal environment occurring at 5100 B.P. during the maximum of the Holocene transgression. As a result, an extensive lagoonal zone was created with a much larger distribution of sand than found at the present day (Caruso Jr., 1992).

\section{MATERIAL AND METHODS}

The material analyzed originates from drilling cores carried out in the southeastern region of the coastal zone of the State of Santa Catarina and designated by prefix LI-01, IM and D. The core LI-01 is located in the central part of the Imaruí Lagoon, $4.5 \mathrm{~km}$ from the coast margin, while IM cores are situated in the district of the municipality of Imaruí, $12 \mathrm{~km}$ from the coast. The core D are located in the D'Una River between Imaruí and Imbituba, about $8 \mathrm{~km}$ from the coast (Figure 1). This study concerns the analysis of 73 samples: 29 samples from the Imaruí area were collected from six sediment-cores (IM-03, IM-06, IM-09, IM-34, IM-40, and IM102) drilled between the SC- 437 highway and the edge of the north of the Imaruí Lagoon; 44 samples from the D'Una River came from eight sediment-cores (D-133, D-200, D-236, D-315, D-323, D-412, and D-434) drilled along the flood plains of the D'Una River; finally nine samples came from the core LI-01 (Figure 1).

The sediment-cores were obtained through the courtesy of the company Inducal, with the aim of defining the calcareous shell reserves and spatial distribution of the beds in the studied localities. Sondeq equipment was used for probing to recover $15 \mathrm{~m}$ of sediments, and the samples were taken at intervals of $60 \mathrm{~cm}$. The core LI-01 was sampled at intervals of $1 \mathrm{~m}$, except for the two superficial samples, which were $5 \mathrm{~m}$ spaced. The material was sieved and dried into three different size fractions: $0.250,0.177$ and $0.074 \mathrm{~mm}$.

The Shannon-Wiener diversity index, equitability and dominance were calculated in level of genera by means of the Paleontological Statistics (PAST) program (Table 1). It is important to record that only the genus Cyprideis is represented by more than one species, C. salebrosa and $C$. multidentata, and both have the same ecological requirements. All the figured specimens (Figure 2, Appendix 1) were illustrated using SEM and they are housed at the Ostracoda section (MP-O) in the collection of the
Departamento de Paleontologia e Estratigrafia of the Universidade Federal do Rio Grande do Sul (UFRGS).

\section{FAUNAL DISTRIBUTION}

The ostracode assemblages studied in both areas are represented by mixohaline species (Cyprideis multidentata, Cyprideis salebrosa, Perissocytheridea kroemmelbeini), eurihaline species (Loxoconcha bullata, Callistocythere litoralensis) and marine species (Orionina similis, Ruggiericythere dimorphica, Argenticytheretta laevipunctata, Neocaudites triplistriatus, Paracypris sp., Cytherella sp. and Bairdopillata sp.). The most abundant mixohaline, euryhaline and marine species are C. multidentata, L. bullata and $R$. dimorphica, respectively (Table 1, Figure 2).

Except by one sample, all the analyzed material from D'Una River and Imaruí show the dominance of $C$. multidentata and C. salebrosa. A low occurrence of L. bullata, $C$. litoralensis, $R$. dimorphica and P. kroemmelbeini it is also recorded, but always more abundant in D'Una River. In both areas instars of $C$. multidentata and C. salebrosa were found even if with a very high A:J ratio. On the contrary, a large number of instars of these two mixohaline species is present in the samples of the LI-01 core (Table 1).

\section{TAPHONOMY AND PALAEOENVIRONMENTS}

Ostracodes are an important tool for making palaeoecological interpretations, inclusive from ancient marginal marine environments. Slack et al. (2000), studying samples from Lake Manzala, considered the most abundant and mixohaline species Cyprideis torosa (Jones) as noise which had masked the signal of rare species. However, our analyses on both D'Una River and Imaruí reveal that the marine species should be considered as noise and the abundant mixohaline Cyprideis as a strong environmental signal. Additional taphonomical and palaeoecological analyses based only on marine taxa, demonstrate that these taxa have an allochthonous population structure and should be considered as environmental noise.

Studies using Recent and Quaternary ostracodes demonstrated that the population age structure analysis of each species included in the ostracod assemblages is useful to discriminate between biocenoses and thanatocenoses (Wagner, 1957; Whatley \& Wall, 1969). This technique has also been used for fossil faunas and leads to recognize the energy levels which characterised the palaeoenvironment (Whatley, 1983, 1988; van Harten 1986; Brouwers, 1988; Whatley \& Boomer, 1995). During their life cycle the ostracodes moults eight times until reaching the adult stage. Thus, in a low energy environment, one individual could leave 18 valves in the sediment. The ideal preservation of the real populational age structure will produce an average of adult:juvenile valves (A:J ratio) of 1:8, where the ninth and last stage is counted as adult. As many early ontogenetic stages are destroyed, mainly by taphocoenotic factors, the A:J ratio will be modified from the ideal $1: 8$ to an interval near $1: 5$ or 1:6, but even intervals 
from 1:3 to 1:6 are considered enough good to characterise authochtonous fossil species (Brouwers, 1988).

The population age structure of Cyprideis multidentata and $C$. salebrosa have been analyzed in this paper using all juveniles and adults collected in the studied cores. In the samples that came from Imaruí and D'Una River the number of adult valves is much higher than the young ones (Table 1 ). According to Brouwers (1988) these high A:J ratios point to an allochthonous concentration deposited in a high energy environment. In the Imaruí and D'Una River cores these species, it is suggested, have suffered post mortem transport processes in high energy environments.

At the LI-O1 core, it was observed that the $C$. multidentata and $C$. salebrosa population age structure is opposite to that shown in Imaruí and D'Una River. In this core, young instars were approximately $80 \%$ of the total population, except for the two first samples, in which the A:J ratio is 1:1 (Table 1). These relatively low ratios could still indicate an allochthonous fauna deposited in high energy environments, but it should be pointed out that the energy level recorded in the LI-01 core should has been lower than that reported in Imaruí and D'Una River because here the A:J ratios are lower. The samples depth 1-2 $\mathrm{m}, 2-3 \mathrm{~m}$ and 6-7 $\mathrm{m}$ present ratios of $1: 2$, showing an asymmetrical proportion of young valves in relation to adult ones. According to Brouwers (1988), this ratio characterizes those faunas that are deposited in low energy environments. The samples depth 3-4 m, 4-5 m, 5-6 $\mathrm{m}$ and 7-8 $\mathrm{m}$ present ratios comprised between 1:3 and 1:6, thus recorded authochtonous fauna, which did not suffer transport post-mortem (Table 1).

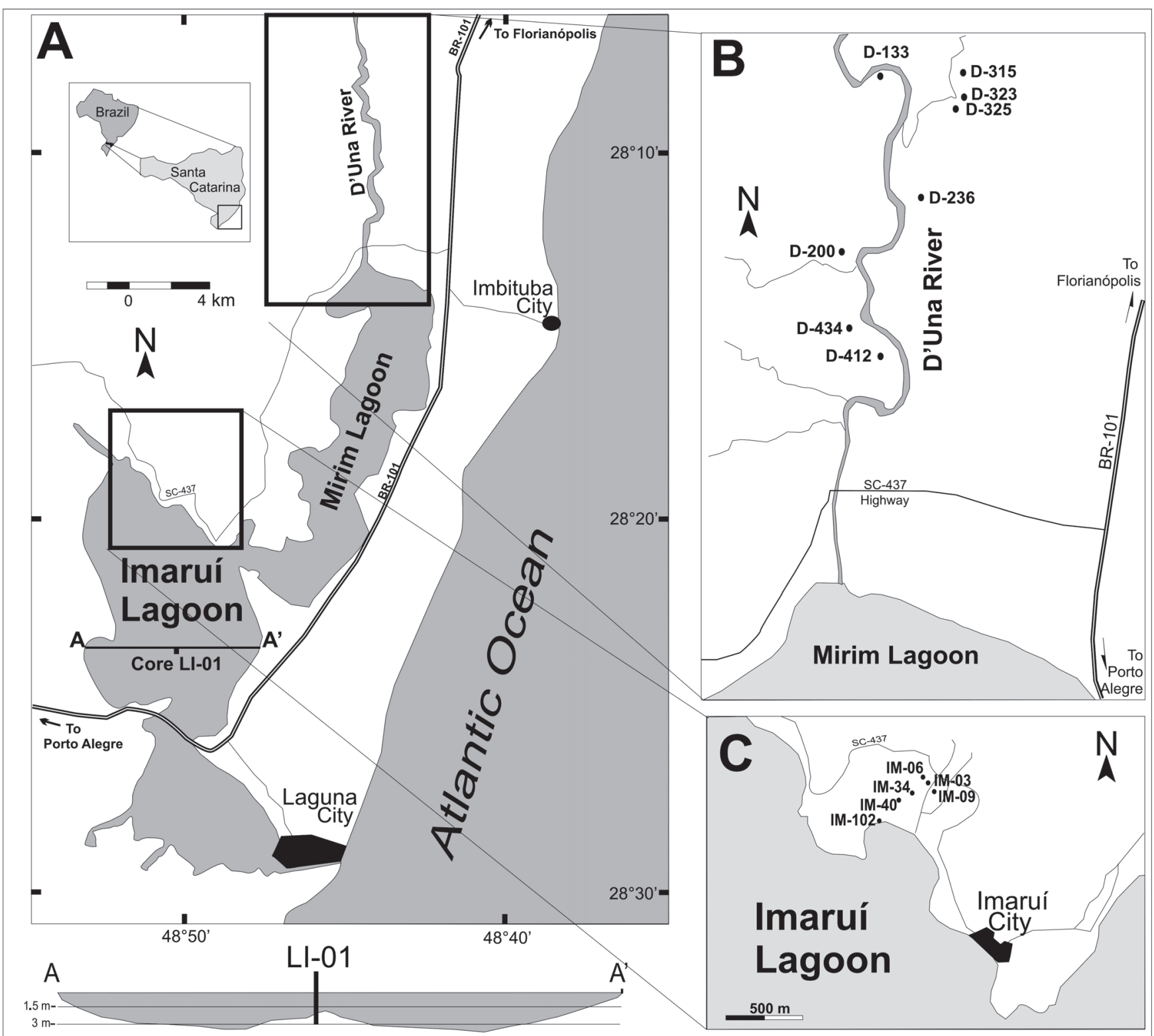

Figure 1. Location map of the study area: A, A-A' transversal section with the core LI-01; B, location of D'Una River area and cores studied; C, location of Imaruí Lagoon and studied cores. 
Table 1. Ecological and sedimentological data and the A:J ratio of Cyprideis in each fossiliferous sample. For the methodology used to calculate the Shannon-Wiener diversity index, equitability and dominance see the text. Total of specimens $=8284 ; \mathrm{OM}=$ organic matter.

\begin{tabular}{|c|c|c|c|c|c|c|c|c|c|c|c|c|c|c|c|c|c|c|c|}
\hline ฏัँ & $\begin{array}{l}\widehat{\Xi} \\
\text { 咅 } \\
\text { 口 }\end{array}$ & 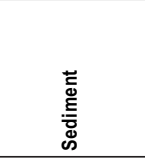 & 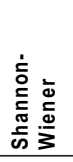 & 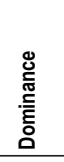 & $\begin{array}{l}\text { 로 } \\
\text { 言 } \\
\text { 䓂 } \\
\text { 豈 }\end{array}$ & 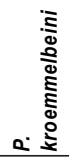 & 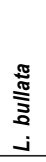 & 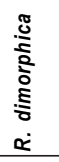 & $\begin{array}{l}\stackrel{0}{*} \\
\text { : } \\
0 \\
0\end{array}$ & 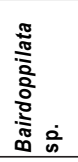 & 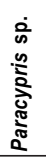 & 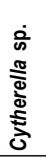 & 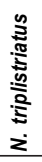 & 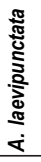 & 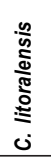 & 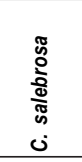 & 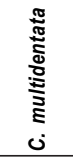 & 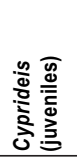 & 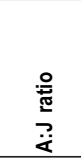 \\
\hline $\begin{array}{l}\text { LII-01 } \\
\text { LI-01 } \\
\text { LI-01 } \\
\text { LI-01 } \\
\text { LI }-01 \\
\text { LI }-01 \\
\text { LI }-01 \\
\text { LI }-01 \\
\text { LI }-01\end{array}$ & $\begin{array}{c}0,0-0,5 \\
0,5-1 \\
1-2 \\
2-3 \\
3-4 \\
4-5 \\
5-6 \\
6-7 \\
7-8 \\
\end{array}$ & $\begin{array}{l}\text { clay } \\
\text { clay } \\
\text { clay } \\
\text { clay } \\
\text { clay } \\
\text { clay } \\
\text { clay } \\
\text { clay } \\
\text { clay } \\
\end{array}$ & $\begin{array}{l}0 . \\
0.31 \\
0.26 \\
- \\
0.04 \\
0.05 \\
0.08 \\
0.65 \\
0.03 \\
\end{array}$ & $\begin{array}{l}1 \\
0.83 \\
0.86 \\
1 \\
0.98 \\
0.98 \\
0.97 \\
0.63 \\
0.99 \\
\end{array}$ & $\begin{array}{l}-\overline{-} \\
0.44 \\
0.38 \\
- \\
0.06 \\
0.04 \\
0.12 \\
0.6 \\
0.05 \\
\end{array}$ & $\begin{array}{l}5 \\
4 \\
\\
1 \\
2 \\
1 \\
\\
1\end{array}$ & $\begin{array}{l}2 \\
1\end{array}$ & & & & & & & & 4 & $\begin{array}{c}2 \\
49 \\
44 \\
67 \\
108 \\
402 \\
46 \\
16 \\
135 \\
\end{array}$ & $\begin{array}{c}6 \\
26 \\
32 \\
152 \\
14 \\
1 \\
49 \\
\end{array}$ & $\begin{array}{c}3 \\
52 \\
111 \\
232 \\
862 \\
2508 \\
225 \\
37 \\
621 \\
\end{array}$ & $\begin{array}{l}01: 01 \\
01: 01 \\
01: 02 \\
01: 02 \\
01: 06 \\
01: 05 \\
01: 03 \\
01: 02 \\
01: 03 \\
\end{array}$ \\
\hline $\begin{array}{l}\mathrm{IM}-03 \\
\mathrm{IM}-03 \\
\mathrm{IM}-03 \\
\mathrm{IM}-03 \\
\mathrm{IM}-03 \\
\end{array}$ & $\begin{array}{c}1,2-1,8 \\
4,2-4,8 \\
4,8-5,4 \\
6,6-7,2 \\
9,6-10,2 \\
\end{array}$ & $\begin{array}{c}\text { clay + OM } \\
\text { clay } \\
\text { clay } \\
\text { clay + OM } \\
\text { clay + OM } \\
\end{array}$ & $\begin{array}{c}- \\
- \\
0.29 \\
0.69 \\
-\end{array}$ & $\begin{array}{c}1 \\
1 \\
0.88 \\
0.5 \\
1 \\
\end{array}$ & $\begin{array}{c}- \\
- \\
0.21 \\
1 \\
- \\
\end{array}$ & 2 & & & & & 1 & 2 & & & $\begin{array}{l}6 \\
2\end{array}$ & $\begin{array}{c}5 \\
29 \\
44\end{array}$ & $\begin{array}{c}17 \\
89 \\
2\end{array}$ & $\begin{array}{c}5 \\
43 \\
27\end{array}$ & $\begin{array}{l}01: 01 \\
01: 01 \\
05: 01\end{array}$ \\
\hline $\begin{array}{l}I M-06 \\
I M-06 \\
I M-06 \\
I M-06 \\
\text { IM-06 } \\
\end{array}$ & $\begin{array}{c}4,8-5,4 \\
5,4-6,0 \\
7,2-7,6 \\
8,4-9,6 \\
9,6-10,8 \\
\end{array}$ & $\begin{array}{l}\text { clay + sand } \\
\text { clay + sand } \\
\text { clay + sand } \\
\text { clay + sand } \\
\text { clay + sand }\end{array}$ & $\begin{array}{c}0 . \\
0.44 \\
0.64 \\
- \\
-\end{array}$ & $\begin{array}{c}1 \\
0.79 \\
0.55 \\
1 \\
1 \\
\end{array}$ & $\begin{array}{c}0 . \\
0.32 \\
0.92 \\
- \\
-\end{array}$ & & 7 & & & & 1 & & 2 & & & $\begin{array}{l}12 \\
27\end{array}$ & $\begin{array}{c}24 \\
53 \\
1 \\
3 \\
3 \\
\end{array}$ & $\begin{array}{c}5 \\
38\end{array}$ & $\begin{array}{l}07: 01 \\
02: 01\end{array}$ \\
\hline $\begin{array}{l}I M-09 \\
I M-09 \\
I M-09 \\
I M-09 \\
\end{array}$ & $\begin{array}{l}3,6-4,2 \\
4,2-4,8 \\
4,8-5,4 \\
6,0-6,6 \\
\end{array}$ & $\begin{array}{l}\text { clay + sand } \\
\text { clay + sand } \\
\text { clay + sand } \\
\text { clay + sand }\end{array}$ & $\begin{array}{c}- \\
- \\
0.16 \\
0.16 \\
\end{array}$ & $\begin{array}{c}1 \\
1 \\
0.93 \\
0.93 \\
\end{array}$ & $\begin{array}{c}- \\
- \\
0.23 \\
0.23 \\
\end{array}$ & & & & & & & & & & $\begin{array}{l}2 \\
2 \\
\end{array}$ & $\begin{array}{c}12 \\
1 \\
4 \\
2 \\
\end{array}$ & $\begin{array}{c}11 \\
3 \\
46 \\
49 \\
\end{array}$ & $\begin{array}{c}7 \\
14 \\
10 \\
\end{array}$ & $\begin{array}{l}03: 01 \\
03: 01 \\
05: 01 \\
\end{array}$ \\
\hline $\begin{array}{l}I M-34 \\
I M-34 \\
I M-34 \\
I M-34 \\
I M-34 \\
I M-34 \\
I M-34 \\
\end{array}$ & $\begin{array}{l}1,2-1,8 \\
1,8-2,4 \\
2,4-3,0 \\
3,0-3,6 \\
3,6-4,2 \\
4,2-4,8 \\
4,8-5,4 \\
\end{array}$ & $\begin{array}{l}\text { clay + sand } \\
\text { clay + sand } \\
\text { clay + sand } \\
\text { clay + sand } \\
\text { clay + sand } \\
\text { clay + sand } \\
\text { clay + sand }\end{array}$ & $\begin{array}{c}- \\
- \\
- \\
0.51 \\
- \\
- \\
-\end{array}$ & $\begin{array}{c}1 \\
1 \\
1 \\
0.68 \\
1 \\
1 \\
1 \\
\end{array}$ & $\begin{array}{c}- \\
- \\
- \\
0.72 \\
- \\
- \\
-\end{array}$ & & $\begin{array}{l}1 \\
1 \\
\end{array}$ & & & & & & & & & $\begin{array}{c}63 \\
13 \\
59 \\
2 \\
1 \\
2\end{array}$ & $\begin{array}{l}1 \\
2 \\
2 \\
3\end{array}$ & $\begin{array}{c}18 \\
5 \\
18\end{array}$ & $\begin{array}{l}04: 01 \\
02: 01 \\
03: 01\end{array}$ \\
\hline $\begin{array}{l}\mathrm{IM}-40 \\
\mathrm{IM}-40 \\
\mathrm{IM}-40 \\
\mathrm{IM}-40 \\
\mathrm{IM}-40 \\
\end{array}$ & $\begin{array}{l}6,0-6,6 \\
6,6-7,2 \\
7,2-7,8 \\
7,8-8,4 \\
8,4-9,0 \\
\end{array}$ & $\begin{array}{l}\text { clay } \\
\text { clay } \\
\text { clay } \\
\text { clay } \\
\text { clay }\end{array}$ & $\begin{array}{c}- \\
- \\
0.45 \\
0.13 \\
- \\
\end{array}$ & $\begin{array}{l}1 \\
1 \\
0.72 \\
0.95 \\
1 \\
\end{array}$ & $\begin{array}{l}- \\
- \\
0.65 \\
0.19 \\
-\end{array}$ & & & & & & & & & & $\begin{array}{l}2 \\
1\end{array}$ & $\begin{array}{c}1 \\
8 \\
2 \\
37 \\
15 \\
\end{array}$ & $\begin{array}{c}1 \\
5 \\
8 \\
44 \\
131 \\
\end{array}$ & $\begin{array}{l}6 \\
6 \\
4 \\
\end{array}$ & $\begin{array}{l}02: 01 \\
13: 01 \\
36: 01 \\
\end{array}$ \\
\hline $\begin{array}{l}I M-102 \\
\text { IM-102 } \\
\text { IM-102 } \\
\text { IM-102 } \\
\text { IM-102 } \\
\end{array}$ & $\begin{array}{l}1,8-2,4 \\
2,4-3,0 \\
3,6-4,2 \\
4,2-4,8 \\
4,8-5,4 \\
\end{array}$ & $\begin{array}{c}\text { clay + OM } \\
\text { clay + OM } \\
\text { clay } \\
\text { clay } \\
\text { clay + OM } \\
\end{array}$ & $\begin{array}{c}- \\
- \\
- \\
- \\
0.69 \\
\end{array}$ & $\begin{array}{c}1 \\
1 \\
1 \\
1 \\
0.5 \\
\end{array}$ & $\begin{array}{l}- \\
- \\
- \\
1 \\
\end{array}$ & & 1 & & & & & & & & & $\begin{array}{c}22 \\
22 \\
1\end{array}$ & $\begin{array}{l}6 \\
3 \\
1 \\
\end{array}$ & 15 & 02:01 \\
\hline $\begin{array}{l}D-133 \\
D-133 \\
D-133 \\
D-133 \\
D-133 \\
D-133 \\
\end{array}$ & $\begin{array}{l}0,6-1,2 \\
1,2-1,8 \\
1,8-2,4 \\
2,4-3,0 \\
3,0-3,6 \\
3,6-4,2 \\
\end{array}$ & $\begin{array}{c}\text { clay } \\
\text { clay }+ \text { sand } \\
\text { clay } \\
\text { clay } \\
\text { clay } \\
\text { clay } \\
\end{array}$ & $\begin{array}{c}- \\
1.06 \\
1.12 \\
- \\
- \\
- \\
\end{array}$ & $\begin{array}{c}1 \\
0.36 \\
0.40 \\
1 \\
1 \\
1 \\
\end{array}$ & $\begin{array}{c}- \\
0.96 \\
0.80 \\
- \\
- \\
- \\
\end{array}$ & & $\begin{array}{l}6 \\
2\end{array}$ & $\begin{array}{l}4 \\
1 \\
2 \\
2 \\
2\end{array}$ & & & & & & & $\begin{array}{l}2 \\
2\end{array}$ & $\begin{array}{l}4 \\
4\end{array}$ & $\begin{array}{l}4 \\
3 \\
1\end{array}$ & 3 & 01:03 \\
\hline $\begin{array}{l}\text { D-200 } \\
\text { D-200 } \\
\text { D-200 } \\
\text { D-200 } \\
\text { D-200 } \\
\text { D-200 } \\
\text { D-200 } \\
\end{array}$ & $\begin{array}{l}1,8-2,4 \\
2,4-3,0 \\
3,0-3,6 \\
3,6-4,2 \\
4,2-4,8 \\
4,8-5,4 \\
5,4-6,0 \\
\end{array}$ & $\begin{array}{l}\text { clay } \\
\text { clay } \\
\text { clay } \\
\text { sand } \\
\text { sand } \\
\text { sand } \\
\text { clay } \\
\end{array}$ & $\begin{array}{l}0.51 \\
0.44 \\
0.99 \\
0.45 \\
0.94 \\
- \\
1.4 \\
\end{array}$ & $\begin{array}{l}0.73 \\
0.79 \\
0.44 \\
0.72 \\
0.43 \\
1 \\
0.3 \\
\end{array}$ & $\begin{array}{l}0.46 \\
0.40 \\
0.71 \\
0.65 \\
0.85 \\
- \\
0.81 \\
\end{array}$ & & $\begin{array}{l}2 \\
4 \\
8 \\
1 \\
7 \\
\end{array}$ & $\begin{array}{c}10 \\
33 \\
5 \\
5 \\
2 \\
\end{array}$ & 4 & & 1 & & 2 & 2 & 2 & $\begin{array}{l}13 \\
12 \\
22\end{array}$ & $\begin{array}{c}51 \\
33 \\
41 \\
1 \\
3 \\
2 \\
11 \\
\end{array}$ & $\begin{array}{l}2 \\
5 \\
9\end{array}$ & $\begin{array}{l}32: 01 \\
09: 01 \\
07: 01\end{array}$ \\
\hline $\begin{array}{l}D-236 \\
D-236 \\
D-236 \\
D-236 \\
D-236 \\
D-236\end{array}$ & $\begin{array}{l}2,4-3,0 \\
3,6-4,2 \\
4,2-4,8 \\
4,8-5,4 \\
6,0-6,6 \\
6,6-7,2 \\
\end{array}$ & $\begin{array}{l}\text { clay } \\
\text { clay } \\
\text { clay } \\
\text { clay } \\
\text { clay } \\
\text { clay }\end{array}$ & $\begin{array}{c}0.19 \\
- \\
0.50 \\
0.50 \\
0.64 \\
- \\
\end{array}$ & $\begin{array}{l}0.91 \\
1 \\
0.68 \\
0.68 \\
0.55 \\
1 \\
\end{array}$ & $\begin{array}{l}0.27 \\
- \\
0.72 \\
0.72 \\
0.99 \\
- \\
\end{array}$ & & 2 & $\begin{array}{l}1 \\
4 \\
1\end{array}$ & & & & & 2 & & & $\begin{array}{l}11 \\
2 \\
2\end{array}$ & $\begin{array}{c}30 \\
9 \\
2 \\
\\
1 \\
\end{array}$ & $\begin{array}{l}3 \\
4\end{array}$ & $\begin{array}{l}14: 01 \\
03: 01\end{array}$ \\
\hline $\begin{array}{l}\text { D-315 } \\
D-315 \\
D-315 \\
D-315 \\
D-315 \\
D-315\end{array}$ & $\begin{array}{l}2,4-3,0 \\
3,0-3,6 \\
3,6-4,2 \\
4,2-4,8 \\
4,8-5,4 \\
5,4-6,0\end{array}$ & $\begin{array}{l}\text { clay } \\
\text { clay } \\
\text { clay } \\
\text { clay } \\
\text { clay } \\
\text { clay }\end{array}$ & $\begin{array}{c}- \\
0.49 \\
0.60 \\
- \\
- \\
-\end{array}$ & $\begin{array}{c}1 \\
0.72 \\
0.60 \\
1 \\
1 \\
1 \\
\end{array}$ & $\begin{array}{c}- \\
0.45 \\
0.86 \\
- \\
- \\
-\end{array}$ & 3 & & $\begin{array}{l}9 \\
5\end{array}$ & & & & & & & & $\begin{array}{c}49 \\
52 \\
2 \\
14 \\
2\end{array}$ & & $\begin{array}{l}2 \\
3\end{array}$ & $\begin{array}{l}25: 01 \\
18: 01\end{array}$ \\
\hline $\begin{array}{l}D-323 \\
D-323 \\
D-323 \\
D-323 \\
\end{array}$ & $\begin{array}{l}3,6-4,2 \\
4,2-4,8 \\
5,4-6,0 \\
7,2-7,8 \\
\end{array}$ & $\begin{array}{l}\text { clay } \\
\text { clay } \\
\text { clay } \\
\text { clay }\end{array}$ & $\begin{array}{l}0.64 \\
0.69 \\
1.02 \\
- \\
\end{array}$ & $\begin{array}{c}0.69 \\
0.50 \\
0.44 \\
1 \\
\end{array}$ & $\begin{array}{l}0.46 \\
0.99 \\
0.73 \\
- \\
\end{array}$ & & $\begin{array}{l}5 \\
9 \\
2 \\
\end{array}$ & $\begin{array}{c}6 \\
11 \\
20\end{array}$ & & & & & & 4 & 2 & $\begin{array}{l}5 \\
2\end{array}$ & $\begin{array}{c}56 \\
9 \\
50\end{array}$ & $\begin{array}{l}6 \\
1 \\
3\end{array}$ & $\begin{array}{l}\text { 10:01 } \\
09: 01 \\
17: 01\end{array}$ \\
\hline $\begin{array}{l}D-325 \\
D-325 \\
D-325 \\
D-325 \\
\end{array}$ & $\begin{array}{l}4,2-4,8 \\
4,8-5,4 \\
5,4-6,0 \\
7,8-8,4 \\
\end{array}$ & $\begin{array}{l}\text { clay } \\
\text { clay } \\
\text { clay } \\
\text { clay }\end{array}$ & $\begin{array}{l}0.86 \\
1.23 \\
0.62 \\
-\quad \\
\end{array}$ & $\begin{array}{l}0.50 \\
0.35 \\
0.63 \\
1 \\
\end{array}$ & $\begin{array}{l}0.79 \\
0.69 \\
0.56 \\
- \\
\end{array}$ & & $\begin{array}{c}10 \\
8 \\
1\end{array}$ & $\begin{array}{c}12 \\
36 \\
8\end{array}$ & 2 & 2 & 1 & & & & 2 & 4 & $\begin{array}{c}40 \\
30 \\
30 \\
3 \\
\end{array}$ & $\begin{array}{l}9 \\
2\end{array}$ & $\begin{array}{l}05: 01 \\
15: 01\end{array}$ \\
\hline $\begin{array}{l}D-412 \\
D-412 \\
D-412 \\
D-412 \\
D-412 \\
D-412 \\
\end{array}$ & $\begin{array}{l}1,2-1,8 \\
1,8-2,4 \\
2,4-3,0 \\
3,0-3,6 \\
3,6-4,2 \\
4,8-5,4 \\
\end{array}$ & $\begin{array}{l}\text { clay } \\
\text { clay } \\
\text { clay } \\
\text { clay } \\
\text { clay } \\
\text { clay }\end{array}$ & $\begin{array}{c}- \\
- \\
0.29 \\
1.02 \\
0.5 \\
-\end{array}$ & $\begin{array}{c}1 \\
1 \\
0.84 \\
0.44 \\
0.68 \\
1 \\
\end{array}$ & $\begin{array}{c}- \\
- \\
0.42 \\
0.73 \\
0.72 \\
- \\
\end{array}$ & & 2 & $\begin{array}{l}4 \\
8 \\
2\end{array}$ & & 2 & & & & & & $\begin{array}{c}49 \\
4 \\
12 \\
5 \\
1 \\
1\end{array}$ & $\begin{array}{c}1 \\
5 \\
30 \\
13 \\
8 \\
4 \\
\end{array}$ & $\begin{array}{l}2 \\
5\end{array}$ & $\begin{array}{l}04: 01 \\
04: 01\end{array}$ \\
\hline \multirow[t]{2}{*}{$\begin{array}{l}D-434 \\
D-434 \\
D-434 \\
D-434 \\
D-434 \\
D-434 \\
\end{array}$} & $\begin{array}{l}2,4-3,0 \\
3,0-3,6 \\
3,6-4,2 \\
4,2-4,8 \\
4,8-5,4 \\
5,4-6,0 \\
\end{array}$ & $\begin{array}{l}\text { clay } \\
\text { clay } \\
\text { clay } \\
\text { clay } \\
\text { clay } \\
\text { clay }\end{array}$ & $\begin{array}{l}- \\
- \\
- \\
- \\
- \\
-\end{array}$ & $\begin{array}{l}1 \\
1 \\
1 \\
1 \\
1 \\
1 \\
\end{array}$ & $\begin{array}{l}- \\
- \\
- \\
- \\
-\end{array}$ & & & & & & & & & & & $\begin{array}{c}34 \\
4 \\
\\
5 \\
5 \\
28 \\
\end{array}$ & $\begin{array}{c}27 \\
12 \\
6 \\
9 \\
25 \\
49 \\
\end{array}$ & $\begin{array}{l}7 \\
3 \\
\\
6 \\
3 \\
4 \\
\end{array}$ & $\begin{array}{l}\text { 09:01 } \\
05: 01 \\
\\
02: 01 \\
10: 01 \\
19: 01 \\
\end{array}$ \\
\hline & & Total & & & & 20 & 83 & 191 & 6 & 4 & 4 & 2 & 6 & 6 & 29 & 1600 & 1386 & 4947 & \\
\hline
\end{tabular}




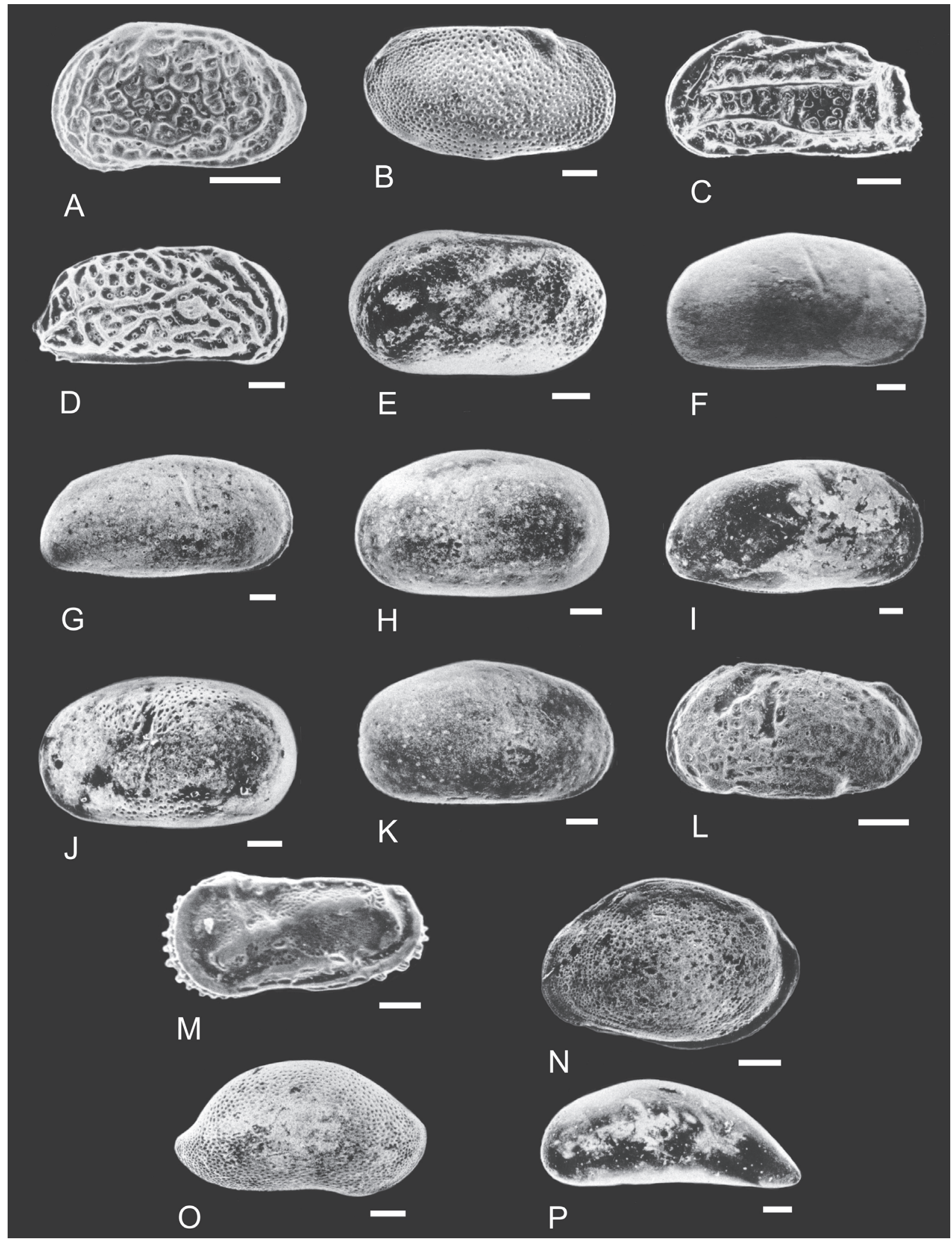

Figure 2. A, Callistocythere litoralensis, MP-O-1976, female carapace left valve; B, Argenticytheretta laevipunctata, MP-O-1977, female right valve; C, Orionina similis, MP-O-1978, left valve; D, Ruggiericythere dimorphica, MP-O-1979, male right valve; E, Cytherella sp., MPO-1980, right valve; F-G, Cyprideis multidentata: F, MP-O-1981, female right valve; G, MP-O-1982, male right valve; H-K, Cyprideis salebrosa: H, MP-O-1983, female left valve; I, MP-O-1984, male right valve; J, MP-O-1985, female left valve; K, MP-O-1986, female right valve; L, Perissocytheridea kroemmelbeini, MP-O-1987, male left valve; M, Neocaudites triplistriatus, MP-0-1988, male left valve; N, Loxoconcha bullata, MP-O-1989, female left valve; O, Bairdoppilata sp., MP-O-1990, right valve; P, Paracypris sp., MP-O-1991, left valve. Scale bar $=100 \mu \mathrm{m}$. 
Studying the micromolluscs in the same region, Mendes (1993) and Pitoni (1993) suggested that the large majority of the studied species were autochthonous. Pitoni (1993) reported that Imaruí's micromolluscs were characteristics of shallow bay or inlet marine environments, with high and low energy areas more influenced by the sea than by the continental processes. On the other hand, based on a detailed geological study, Caruso Jr. (1995b) proposed another origin of the calcareous shells found in this region, to which the ostracodes and micromolluscs are associated, suggesting that the region was characterized by a large lagoonal body formed during the maximum Holocene transgression. It is pointed out that the micromollusc shells are accumulated and concentrated by high energy processes due to the presence of levels with gradational layering and a mixing of lagoonal mixohaline and marine fauna. According to Caruso Jr. (1995b, 1999), the intensity of the winds over the lagoons was able to produce waves that generate coastal currents eroding and depositing sediments along their margins, reworking old deposits, generating beaches, and building sandy areas.

\section{DISCUSSION AND CONCLUSIONS}

Mendes (1993) and Pitoni (1993), recorded specimens that inhabit modern shallow marine, high and low energy environments, associated with species typical of estuaries and lagoons. According to those authors, the majority of these species present a balanced population age structure typical of an authochtonous fauna. According to Mendes (1993), the dominant species of marine gastropods and bivalves are constituted mainly by fragmented, rolled and young shells (e.g. Finella dubia (d'Orbigny, 1842); Mytilidae). Notwithstanding these data, Mendes (1993) and Pitoni (1993), consider that marine and lagoonal micromollusc faunas did not suffer post-mortem transport, suggesting that they were deposited in a shallow marine environment under some estuarine or lagoonal influence.

Taking into account the ecological and palaeoecological preferences of $C$. multidentata and C. salebrosa, typically found in mixohaline environments, it is possible to assume that the environment where these species lived was probably a marginal marine lagoon with some mixohaline characteristics, because living specimens of $C$. multidentata and C. salebrosa have a low tolerance to normal marine salinity. Whatley (1988) records that mixohaline ostracode faunas possess low diversities and high dominances. These characteristics are observed in the Imaruí, D'Una River and LI-01 samples by the majority of the assemblages (Table 1). The shallow marine (A. laevipunctata, Bairdoppilata sp., Cytherella sp., $N$. triplistriatus, $O$. similis, Paracypris sp. and $R$. dimorphica) and eurihaline ( $C$. litoralensis and $L$. bullata) species are represented only by very few adult specimens. Anyway, high energy environments are suggested for the Imaruí and D'Una River palaeolagoons (mainly ostracode adult valves) and, partially, also for the central portion of the Imaruí Lagoon (LI01 core) in which only few samples (core depths: $3-4 \mathrm{~m}, 4-5 \mathrm{~m}$,
5-6 $\mathrm{m}$ and 7-8 $\mathrm{m}$ ) recorded autochthonous ostracode assemblages. These conclusions are in agreement with Caruso Jr. (1995b, 1999) statement that the regional calcareous shell deposits were formed by high energy environmental processes.

Thus, the ostracode data have provided a very different palaeoenvironmental interpretation from those drawn from micromolluscs. In fact, the present study suggests that the marine ostracodes were moved, after death, into the lagoonal system, as no juvenile ontogenetic stages of these species were found and the adults are represented by few specimens (Table 1). Morover, the mixohaline ostracode fauna which lived in a lagoonal environment, underwent post-mortem transport into the lagoon itself, being reworked together with the marine ostracodes, and then redeposited.

Based on the above data and the sedimentary structures observed by Caruso Jr. (1995a, 1999), it is assumed that the ostracodes and micromolluscs, typical of shallow marine environments, were transported inside the lagoons through the action of storms and tides more than by the relative sea level oscillations. Inside the lagoons lived an autochthonous fauna of mixohaline micromolluscs and ostracodes. The wind action generated waves and currents inside the lagoons, that were able to rework the pre-existent deposits allowing the fragmentation of the shells, thus mixing faunas from different environments, and redepositing this material on the lagoon banks.

\section{ACKNOWLEDGMENTS}

The present study largely benefits from the cores provided by CECO (Centro de Estudos Costeiros e Oceanográficos) at UFRGS. JCC gratefully acknowledges the CNPq by the financial support (grants 520309/99-5 and 475313/03-8). During an early stage of the project, Francisco Caruso Jr., Yvonne T. Sanguinetti and Felipe Toledo made valuable suggestions. We are also deeply indebted to Elsa Gliozzi and Stephen Eagar for their critical review and suggestions which greatly improved the manuscript.

\section{REFERENCES}

Brouwers, E.M. 1988. Sediment transport detected from analysis of ostracod population structure: an example from the Alaska continental shelf. In: P. de Decker, J.P. Colin \& J.P. Peypouquet (eds.) Ostracoda in the Earth Sciences, Elsevier, p. 231-244.

Carreño, A.L.; Coimbra, J.C. \& do Carmo, D.A. 1999. Late Cenozoic sea level changes evidenced by ostracods in the Pelotas Basin, southernmost Brazil. Marine Micropaleontology, 37:117-129.

Caruso Jr., F. 1992. Geologia dos depósitos de conchas calcárias no Estado de Santa Catarina. Geosul, 14:101-136.

Caruso Jr., F. 1995a. Complexo lagunar do Mirim, Imaruí e Santo Antônio (SC): aspectos morfológicos e sedimentológicos do fundo lagunar. In: CONGRESSO DA ASSOCIAÇÃO BRASILEIRA DE ESTUDOS DO QUATERNÁRIO, 5, 1995. Anais, Niterói, UFF, p. 81-87. 
Caruso Jr., F. 1995b. Geologia e recursos minerais da região costeira do sudeste de Santa Catarina com ênfase no Cenozóico. Programa de Pós-Graduação em Geociências, Universidade Federal do Rio Grande do Sul, PhD. Dissertation, 179 p.

Caruso Jr., F. 1999. Shell deposits in the Santa Catarina coastal area, southern region of Brazil. In: L.R. Martins \& C.I. Santana (eds.) Non living resources of the southern Brazilian coastal zone and continental margin, UNESCO/Editora da UFRGS, p. 69-79.

Caruso Jr., F.; Suguio, K. \& Nakamura, T. 1999. The Quaternary geological history of Santa Catarina southeastern region (Brazil). Anais da Academia Brasileira de Ciências, 72(2):257-270.

Gré, J.C.R.; Klingebiel, A.; Horn, N.O. \& Caruso Jr., F. 1993. Morphologie, structure et evolution du cadre géologique du systeme lagunaire Santo Antonio, Etat de Santa Catarina, Brésil. Bulletin de l'Institut Géologique du Bassin d'Aquitaine, 53(1):159-167.

van Harten, D. 1986. Use of ostracodes to recognize downslope contamination in paleobathymetry and a preliminary reappraisal of the paleodepth of the Prasás Marls (Pliocene), Crete, Greece. Geology, 14:856-859.

Hartmann, G. \& Puri, H.S. 1974. Summary of neontological and paleontological classification of Ostracoda. Mitteilung Zoologisches Museum Institut, 70:7-73.

Horne, D.; Cohen, A. \& Martens, K. 2002. Taxonomy, morphology and biology of Quaternary and living Ostracoda. In: J.A. Holmes \& A. Chivas (eds.) The Ostracoda: applications in Quaternary research, Washington, American Geophysical Union, p. 5-36 (Geophysical Monograph 131).

Martin, L. \& Suguio, K. 1986. Excursion route along the coastal plains of the states of Paraná and Santa Catarina. In: INTERNATIONAL SYMPOSIUM ON SEA LEVEL CHANGES AND QUATERNARY SHORELINES, 1, 1986. Field guide, São Paulo, USP, p. 1-124.

Martin, L.; Suguio, K.; Flexor, J.M. \& Azevedo, A.E.G. 1988. Mapa geológico do Quaternário costeiro dos estados do Paraná e Santa Catarina. Rio de Janeiro, Departamento Nacional da Produção Mineral, Divisão de Geologia e Mineralogia, 40 p. (Série Geologia, 28, Seção Geologia Básica, 18).

Mendes, I.L.V. 1993. Malacofauna, paleoecologia e bioestratigrafia de sedimentos holocênicos da planície costeira de Imbituba e Imaruí, Santa Catarina, Brasil. Programa de Pós-Graduação em Geociências, Universidade Federal do Rio Grande do Sul, Ph.D. Dissertation, 309 p.

Pitoni, V.L.L. 1993. Moluscos cenozóicos de sub-superfície em Imaruí, Santa Catarina, Brasil: paleoecologia, transgressões e regressões. Programa de Pós-Graduação em Geociências, Universidade Federal do Rio Grande do Sul, PhD. Dissertation, 269 p.

Slack, J.M.; Kaesler, R.L. \& Kontrovitz, M. 2000. Trend, signal and noise in the ecology of Ostracoda: information from rare species in low-diversity assemblages. Hydrobiologia, 419:181-189.

Suguio, K.; Angulo, R.J.; Carvalho, A.M.; Corrêa, I.C.S.; Tomazelli, L.J.; Willwock, J.A. \& Vital, H. 2005. Paleoníveis do mar e paleolinhas de costa. In: C.R.G. Souza; K. Suguio; A.M.S. Oliveira \& P.E. Oliveira (eds.) O Quaternário do Brasil. Associação Brasileira de Estudos do Quaternário, p. 130-152.

Suguio, K.; Martin, L.B.; Bittencourt, A.C.S.P.; Dominguez, J.M.L. \& Azeredo, A.E.G. 1985. Flutuações do nível relativo do mar durante o Quaternário superior ao longo do litoral brasileiro e suas implicações na sedimentação costeira. Revista Brasileira de Geociências, 15(4):273-286.

Thiesen, Z.V.; Corbelini, L.M.; Mendes, I.L.V. \& Pitoni, V.L.L. 1993. Contribuição ao estudo paleoambiental da região de
Imbituba e Imaruí, Santa Catarina-Foraminíferos. In: CONGRESSO BRASILEIRO DE PALEONTOLOGIA, 13, 1993. Livro de Resumos, São Leopoldo, UNISINOS, p. 77.

Villwock, J.A. \& Tomazelli, L.J. 1995. Geologia costeira do Rio Grande do Sul. Notas Técnicas, 8:1-45.

Wagner, C.W. 1957. Sur les Ostracodes du Quaternarie Récent des Pays-Bas et leur utilisation dans l'Étude Géologique des Depôts Holocènes. Faculté des Sciences, Université de Paris, PhD. Thesis, $259 \mathrm{p}$.

Whatley, R.C. 1983. The application of Ostracoda to palaeoenviromental analysis. In: R.F. Maddocks (ed.) Applications of Ostracoda, University of Houston Press, p. 51-77.

Whatley, R.C. 1988. Ostracoda and Palaeogeography. In: P. De Decker; J.P. Colin \& J.P. Peypouquet (eds.) Ostracoda in the Earth Sciences, Elsevier, p. 103-123.

Whatley, R.C. \& Boomer, I. 1995. Autochthonous and allochthonous Quaternary Ostracoda from ODP Site 893, Santa Barbara Basin. In: J.P. Kennett; J.G. Baldauf \& M. Lyle (eds.) Proceedings of the Ocean Drilling Program, Houston, p. 251-255 (Scientific Results 146).

Whatley, R.C. \& Wall, D.R. 1969. A preliminary account of the ecology and distribution of Recent Ostracoda in the South Irish Sea. In: J.W. Neale (ed.) Taxonomy, morphology and ecology of Recent Ostracoda, Oliver and Boyd, p. 268-288.

Received in June, 2006; accepted in October, 2006

Appendix 1. Ostracoda species examined in this study. We follow Moore \& Pritat (1961) in considering the traditional classification of Ostracoda to be a suitable option for the present, but note that some authors follow the supra-generic categories modified by Horne et al. (2002). However, since Hartmann \& Puri (1974) the genus Paracypris Sars, 1866 has been included within the family Candonidae Kaufmann, 1900 as adopted below.

Suborder Platycopina Sars, 1866

Family Cytherellidae Sars, 1866

Genus Cytherella Jones, 1849

Cytherella sp.

Suborder Podocopina Sars, 1866

Superfamily Cytheracea Bair, 1850

Family Leptocytheridae Hanai, 1957

Genus Callistocythere Ruggieri, 1953

Callistocythere litoralensis (Rossi de García, 1966) emend. Sanguinetti, Ornellas \& Coimbra, 1991

Family Cytherettidae Triebel, 1952

Genus Argenticytheretta Rossi de García, 1969 emend. Sanguinetti, Ornellas \& Coimbra, 1991

Argenticytheretta laevipunctata Sanguinetti, Ornellas \& Coimbra, 1991 Family Hemicytheridae Puri, 1953

Genus Orionina Puri, 1954 emend. Coimbra \& Ornellas, 1986 Orionina similis Bold, 1963 emend. Coimbra \& Ornellas, 1986

Genus Ruggiericythere Aiello, Coimbra \& Barra, 2004

Ruggiericythere dimorphica (Whatley et al., 1998) emend. Aiello, Coimbra \& Barra, 2004

Family Cytherideidae Sars, 1925

Genus Cyprideis Jones, 1857

Cyprideis multidentata Hartmann, 1955

Cyprideis salebrosa Bold, 1963

Genus Perissocytheridea Stephenson, 1938 emend. Pinto \& Ornellas, 1970

Perissocytheridea kroemmelbeini Pinto \& Ornellas, 1970 
Family Trachyleberididae Sylvester-Bradley, 1948

Genus Neocaudites Puri, 1960

Neocaudites triplistriatus (Edwards, 1944) emend. Bold, 1963

Family Loxoconchidae Sars, 1925

Genus Loxoconcha Sars, 1866

Loxoconcha bullata Hartmann, 1956

Superfamily Bairdiacea Sars, 1888

Family Bairdiidae Sars, 1888

Genus Bairdoppilata Coryell, Sample \& Jennings, 1935

Bairdoppilata sp.

Superfamily Cypridacea Baird, 1845

Family Candonidae Kaufmann, 1900

Subfamily Paracyprididae Sars, 1923

Genus Paracypris Sars, 1866

Paracypris sp. 